\title{
Mixed-mode fatigue crack growth behaviour in aluminium alloy
}

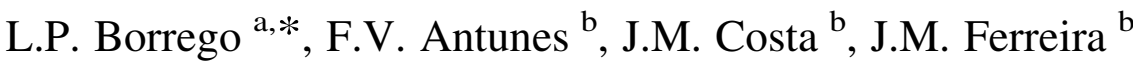 \\ ${ }^{a}$ Department of Mechanical Engineering, ISEC, Rua Pedro Nunes, Quinta da Nora, 3030-199 Coimbra, Portugal \\ ${ }^{\mathrm{b}}$ Department of Mechanical Engineering, University of Coimbra, Polo II, Pinhal de Marrocos, 3030-201 Coimbra, Portugal,
}

Received in revised form 29 June 2005; accepted 6 July 2005

Available online 15 December 2005

\begin{abstract}
Fatigue crack propagation tests in compact mixed-mode specimens were carried out for several stress intensity ratios of mode I and mode II, $K_{\mathrm{I}} / K_{\mathrm{II}}$, in AlMgSi1-T6 aluminium alloy with $3 \mathrm{~mm}$ thickness. The tests were performed in a standard servo-hydraulic machine. A linkage system was developed in order to permit the variation of the $K_{\mathrm{I}} / K_{\mathrm{II}}$ ratio by changing the loading angle. Crack closure loads were obtained through the compliance technique. A finite element analysis was also done in order to obtain the $K_{\mathrm{I}}$ and $K_{\mathrm{II}}$ values for the different loading angles. Crack closure increases under mixed-mode loading conditions in comparison to mode-I loading due the friction between the crack tip surfaces. Moreover, the crack closure level increases with the $K_{\mathrm{I}} / K_{\mathrm{II}}$ ratio decrease. Correlations of the equivalent values of the effective stress intensity factor with the crack growth rates are also performed. Finally, an elastic-plastic finite element analysis was performed to obtain the plastic zones sizes and shapes and model the effect of mixed-mode loading on crack closure.
\end{abstract}

(C) 2005 Elsevier Ltd. All rights reserved.

Keywords: Aluminium alloy; Mixed-mode loading; Fatigue crack propagation; Crack closure

\section{Introduction}

Age hardened aluminium alloys are of great technological importance. In particular for ground transport systems, when relatively high strength, good corrosion resistance and high toughness are required in conjunction with good formability and weldability, aluminium alloys with $\mathrm{Mg}$ and $\mathrm{Si}$ as alloying elements are used ( $\mathrm{Al}-\mathrm{Mg}-\mathrm{Si} / 6 \mathrm{xxx}$ series).

The majority of the fatigue crack growth studies are usually performed under mode-I loading conditions. However, in many practical cases cracks are not normal to the maximum principal stress direction, and a mixed-mode (combined modes I and II) condition prevails at the tip of such cracks. This type of loading is very commonly encountered when crack deflection occurs during crack growth (that happens near-threshold) or when crack branching occurs. Although analytical and experimental studies have also been extensively conduced under mixedmode loading (ex. [1-4]) the amount of available data related to the crack closure behaviour under this type of loading is reduced [5].

\footnotetext{
* Corresponding author. Tel.: +351 239790 700; fax: +351 239790331.

E-mail address: borrego@isec.pt (L.P. Borrego).
}

0142-1123/\$ - see front matter (C) 2005 Elsevier Ltd. All rights reserved. doi:10.1016/j.ijfatigue.2005.07.047
Crack closure is the contact of crack flanks during a portion of the load cycle. It delays the intrinsic mechanisms responsible for crack growth (cyclic plastic deformation, clivage, etc), therefore it must be considered in the modelling of fatigue crack growth. In fact, crack closure seems to be able to explain, among other aspects, the influence of mean stress [6-8], microstructure $[9,10]$ and the transient crack growth behaviour following overloads [8-11]. Crack closure is mainly induced by crack wake plasticity, by the presence of oxides and by the roughness of fracture surfaces. The plasticity-induced closure mechanism is dominant at medium and high $\Delta K$ values, while the other mechanisms are more important at low values of $\Delta K$ (near-threshold).

Crack closure induced by plasticity can be studied using experimental or numerical approaches. Experimental studies have played a major role in the understanding of this phenomenon. The numerical approach is also interesting to study crack closure, because once the numerical procedure is optimised, it is relatively simple to adapt it to new load conditions, materials, crack lengths, etc. However, the finite element models must be correctly defined and their limitations understood [12].

In recent work the authors [8] concluded that crack closure was able to explain the influence of the stress ratio on the fatigue crack growth rate under mode-I loading for the AlMgSi1-T6 aluminium alloy in both Paris regimes and near threshold. Furthermore, also for mode-I, the plasticity-induced crack closure phenomenon could generally explain the crack 
growth behaviour following single peak overloads under both load control mode [8] and constant $\Delta K$ conditions [11]. Present work intends to analyse the fatigue crack growth under mixedmode (mode-I and mode-II) and the correspondent crack closure behaviour.

\section{Experimental procedure}

This research was conduced using the AlMgSi1 (6082) aluminium alloy with a T6 heat treatment. The T6 treatment is a full heat treatment process comprising the operations of solution treatment, quenching and age-hardening. The chemical composition and the mechanical properties of the alloy are shown in Tables 1 and 2, respectively.

Mixed-mode (mode-I and mode-II) fatigue crack growth tests were conducted on Compact Tension Shear (CTS) specimens with a thickness of $3 \mathrm{~mm}$. The specimens were obtained in the transverse longitudinal (TL) direction from a laminated plate. The initial notch depth was $42.5 \mathrm{~mm}$. Fig. 1 illustrates the major dimensions of the samples used in the tests. A main geometrical parameter depicted in this figure is the slope at the crack tip, $\beta$, under mixed-mode load. Before testing the specimen surfaces were polished mechanically.

The testes were performed using the loading device shown in Fig. 2. This apparatus was based on the mixed-mode fracture testing technique originally developed by Richard [13]. The loading device allows to apply pure mode-I, pure mode-II, as well as mixed-mode loading to the CTS specimen just by changing the loading angle $\alpha$ between the longitudinal axis of the specimen and the load direction applied by an uniaxial tension testing machine.

The specimen has circular holes while the loading device has elongated holes. External holes are elongated in the direction parallel to the crack so that forces are normal to the initial mode-I crack plane. On the other hand, middle holes are elongated perpendicular to the crack so that only the forces parallel to the notch and the initial mode-I crack can be transmitted from the load device to the specimen (see Fig. 1).

The experiments were performed in a servohydraulic, closed-loop mechanical test machine with $100 \mathrm{kN}$ load

Table 1

Chemical composition of AlMgSi1-T6 aluminium alloy [wt\%]

\begin{tabular}{lllllllll}
\hline $\mathrm{Si}$ & $\mathrm{Mg}$ & $\mathrm{Mn}$ & $\mathrm{Fe}$ & $\mathrm{Cr}$ & $\mathrm{Cu}$ & $\mathrm{Zn}$ & $\mathrm{Ti}$ & other \\
\hline 1.05 & 0.80 & 0.68 & 0.26 & 0.01 & 0.04 & 0.02 & 0.01 & 0.05 \\
\hline
\end{tabular}

Table 2

Mechanical properties of AlMgSi1-T6 alloy

\begin{tabular}{ll}
\hline Tensile strength, $\sigma_{\mathrm{UTS}}[\mathrm{MPa}]$ & $300 \pm 2.5$ \\
Yield strength, $\sigma_{\mathrm{YS}}[\mathrm{MPa}]$ & $245 \pm 2.7$ \\
Elongation, $\varepsilon_{\mathrm{r}}[\%]$ & 9 \\
Cyclic hardening exponent, $n^{\prime}$ & 0.064 \\
Cyclic hardening coefficient, $K^{\prime}[\mathrm{MPa}]$ & 443 \\
Fatigue strength exponent, $b$ & -0.0695 \\
Fatigue strength coefficient, $\sigma^{\prime}{ }_{\mathrm{f}}[\mathrm{MPa}]$ & 485 \\
Fatigue ductility exponent, $c$ & -0.827 \\
Fatigue ductility coefficient, $\varepsilon^{\prime}$ & 0.773
\end{tabular}

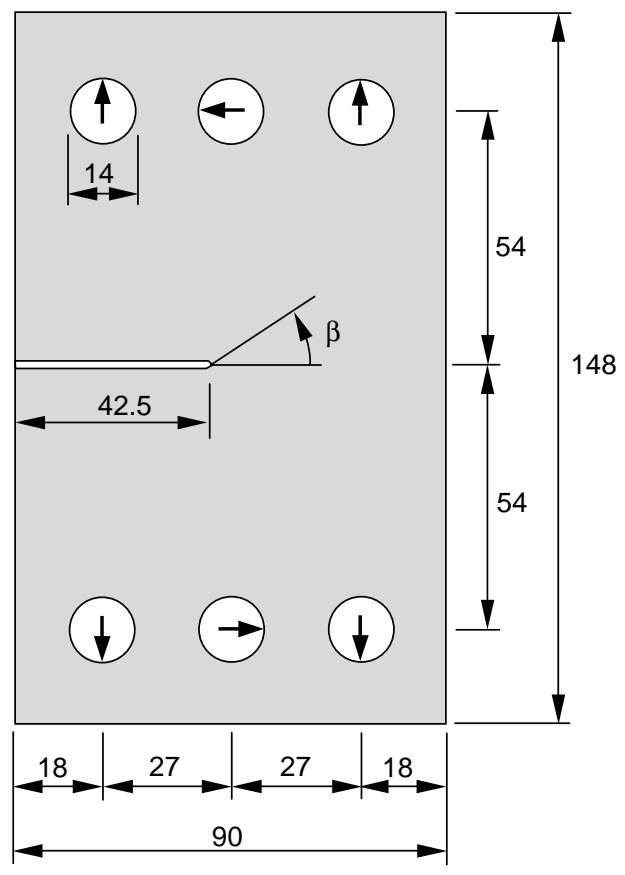

Fig. 1. Geometry of the CTS specimen used in this work (dimensions in mm).

capacity, interfaced to a computer for machine control and data acquisition. All tests were conducted in air and at room temperature. The tests were performed in load control mode and the load ratio for all loading angles was kept constant at 0.05 . The loads were applied with a sinusoidal waveform at a frequency of $20 \mathrm{~Hz}$. Fatigue pre-cracking was introduced under

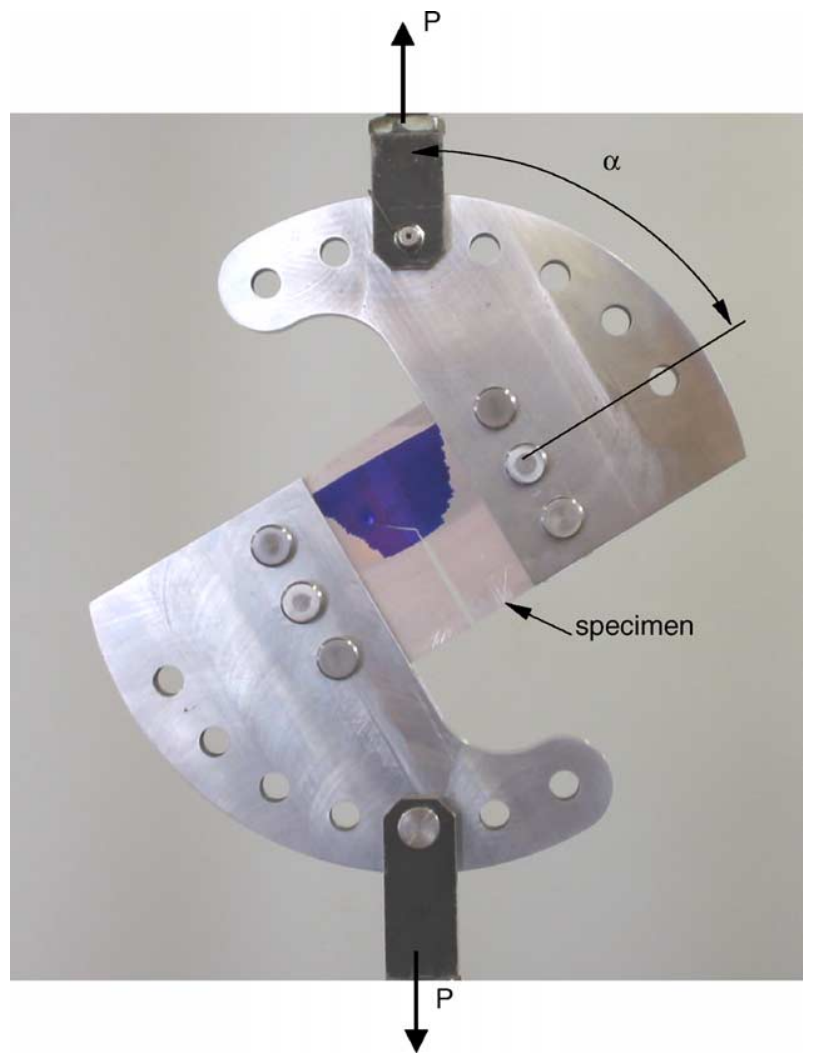

Fig. 2. Mixed-mode Loading device $\left(\alpha=60^{\circ}\right)$. 
mode-I loading to an $a / W$ ratio of 0.51 , where $a$ and $W$ are the crack length and width of the specimen, respectively. The crack length was measured in both $x$ and $y$ directions using a travelling microscope $(45 \times)$ with a resolution of $10 \mu \mathrm{m}$. The specimen was painted ahead of the crack tip for enhance optical measurement of crack length (see Fig. 2).

Several loading angles were analysed, namely, $\alpha=0,30,45$ and $60^{\circ}$. The influence of the different mixed-mode loadings was investigated in the Paris regime. The crack growth rates were determined by the incremental polynomial method using five consecutive points.

Load-displacement behaviour was monitored at specific intervals throughout each of the tests using a pin microgauge elaborated from a high sensitive commercial axial extensometer $( \pm 0.625 \mathrm{~mm}$ of maximum displacement $)[10]$. The gauge pins were placed in two drilled holes of $0.5 \mathrm{~mm}$ diameter located at the centre of the specimen, above and below the mode-I loading crack plane. The distance between these holes was $3.5 \mathrm{~mm}$. The pin microgauge was always orientated accordingly to the external loading direction as illustrated in Fig. 3. In order to collect as many load-displacement data as possible during a particular cycle, the frequency was reduced to $0.5 \mathrm{~Hz}$. Noise on the strain gauge output was reduced by passing the signal through a $1 \mathrm{~Hz}$ low-pass mathematical filter.

From the load-displacement records, variations of the opening load, $P_{\mathrm{op}}$, were derived using the technique known as maximisation of the correlation coefficient [14]. This technique involves taking the upper $10 \%$ of the $P-\varepsilon$ data and calculating the least squares correlation coefficient. The next data pair is then added and the correlation coefficient is again computed. This procedure is repeated for the whole data set. The point at which the correlation coefficient reaches a maximum could then be defined as $P_{\mathrm{op}}$.

The fraction of the load cycle for which the crack remains fully open, parameter $U$, was calculated by the following equation:

$U=\frac{P_{\max }-P_{\mathrm{op}}}{P_{\max }-P_{\min }}$

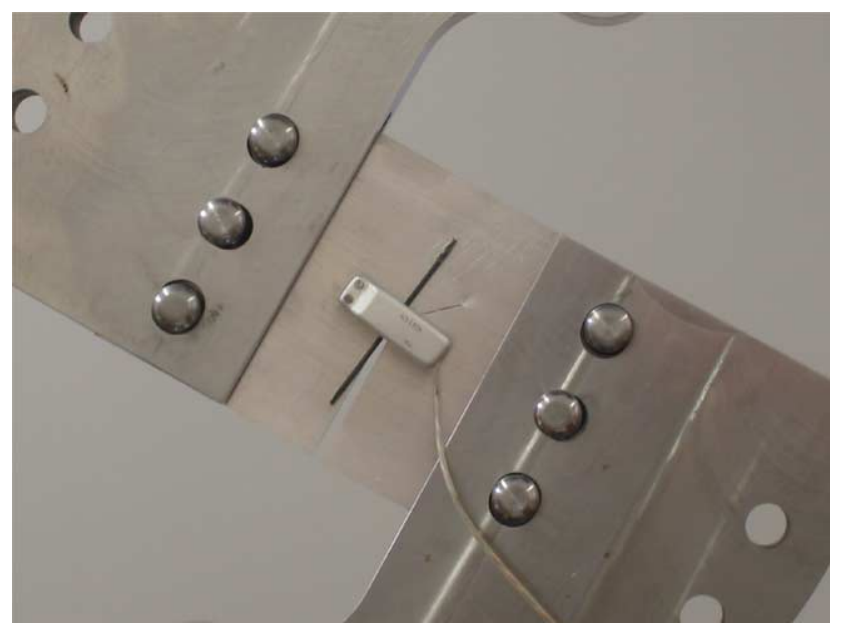

Fig. 3. Pin gauge location $\left(\alpha=60^{\circ}\right)$. where $P_{\max }, P_{\min }$, and $P_{\text {op }}$ are the maximum, minimum and crack opening loads, respectively.

The accuracy of the all process used to obtain the crack closure level, as well as the sensitivity of pin gauge location with respect to the crack tip were evaluated under mode I loading in ref. [10]. The pin gauge was placed at the centre line of the specimen, at $3 \mathrm{~mm}$ behind the crack tip and at $1 \mathrm{~mm}$ behind the crack tip. Three load-displacement records were obtained for each position of the pin gauge. It was shown that the obtained crack closure levels are fairly accurate, with variations of parameter $U$ lower than $1 \%$ in all cases. Furthermore, for distances higher than $1 \mathrm{~mm}$, the crack closure values were practically independent of the pin gauge location relatively to the crack tip.

\section{Stress intensity factors for CTS specimen}

Richard [13] obtained $K_{I}$ and $K_{I I}$ solutions for the CTS geometry considering a central crack, plane and normal to lateral faces. The stress intensity factors for different loading angles and the original crack orientation are given by the following expressions:

$$
\begin{gathered}
K_{\mathrm{I}}=\frac{F}{W B} \sqrt{\pi a} \frac{\cos \alpha}{\left(1-\frac{a}{W}\right)} \sqrt{\frac{0.26+2.65\left(\frac{a}{W-a}\right)}{1+0.55\left(\frac{a}{W-a}\right)+0.08\left(\frac{a}{W-a}\right)^{2}}} \\
K_{\mathrm{II}}=\frac{F}{W B} \sqrt{\pi a} \frac{\sin \alpha}{\left(1-\frac{a}{W}\right)} \sqrt{\frac{-0.23+1.40\left(\frac{a}{W-a}\right)}{1-0.67\left(\frac{a}{W-a}\right)+2.08\left(\frac{a}{W-a}\right)^{2}}}
\end{gathered}
$$

where $F$ is the applied force, $W$ is the width of the specimen, $B$ is the thickness of the specimen, $a$ is the crack length and $\alpha$ is the angle of loading direction with respect to the crack plane. The degree of the mode-mixity is given by:

$M=\frac{2}{\pi} a \tan \left(\frac{K_{\mathrm{I}}}{K_{\mathrm{II}}}\right)$

These stress intensity factor solutions are adequate for fracture studies, since pre-cracks are obtained under mode-I loading, therefore do not suffer crack deflection. However, cracks submitted to mixed mode fatigue loading change orientation searching mode-I loading, thus Eqs. (3) and (4) are inadequate, since they have been developed for the initial crack orientation, i.e. straight cracks. Therefore, a numerical analysis was performed in order to obtain both $K_{I}$ and $K_{I I}$ stress intensity factors under mixed-mode fatigue loading.

Several crack geometries were studied numerically by the finite element method and solutions were fitted to numerical predictions. A 2D analysis along with a plane strain state were assumed. Due to lack of symmetry in terms of loading and geometry of the crack, the whole specimen was analysed. The boundary conditions considered are presented in Fig. 4, and intend to avoid rigid body movement without affecting the global rigidity of the specimen. The material was assumed to be homogeneous, isotropic with linear elastic behaviour. The 


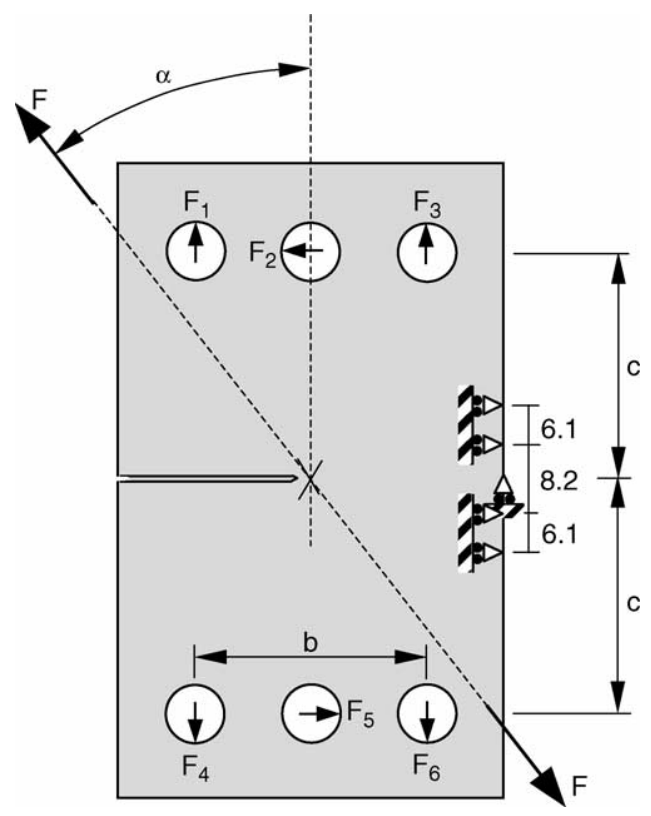

Fig. 4. Loading and boundary conditions for CTS specimen.

elastic properties considered were $E=74000 \mathrm{MPa}$ and $\nu=$ 0.33 .

The uniaxial load $F$ is related with punctual loads according to the following expressions [13]

$F_{1}=F_{6}=F\left(\frac{1}{2} \cos \alpha+\frac{c}{b} \sin \alpha\right)$

$F_{2}=F_{5}=F \sin \alpha$

$F_{3}=F_{4}=F\left(\frac{1}{2} \cos \alpha-\frac{c}{b} \sin \alpha\right)$

The physical model was analysed using CosmosM 2.0 [15], a commercial finite element package. Quadrilateral isoparametric elements with eight nodes were used. Singular elements with nodes at quarter-point positions were considered at the crack tip. Fig. 5 presents meshes considered for different slopes at the crack tip (denoted $\beta$ in Fig. 1), being the crack tip at $52.5 \mathrm{~mm}$ and at the notch plane $(y=0)$. The meshes had 4804 elements and 15075 nodes. It is important to notice that the geometry of crack remote from its tip does not influence $K$ values (see Fig. 5(b)). The accuracy of numerical predictions was checked by comparison with Eq. (3) and Eq. (4) for $\beta=0^{\circ}$ and for several loading angles. Differences lower than $2 \%$ were obtained for $K_{\mathrm{I}}$ and $K_{\mathrm{II}}$ which is a good indication for results accuracy.

Several numerical analysis were performed (1120) considering 32 crack tip locations, seven loading angles, $\alpha$, and five different slops at the crack tip, $\beta$. A numerical solution with 39 parameters was fitted to the numerical results. The stress intensity factors $K_{\mathrm{I}}$ and $K_{\mathrm{II}}$ for all test conditions were then calculated from these solutions obtained for the CTS specimen under mixed-mode fatigue loading. (a)

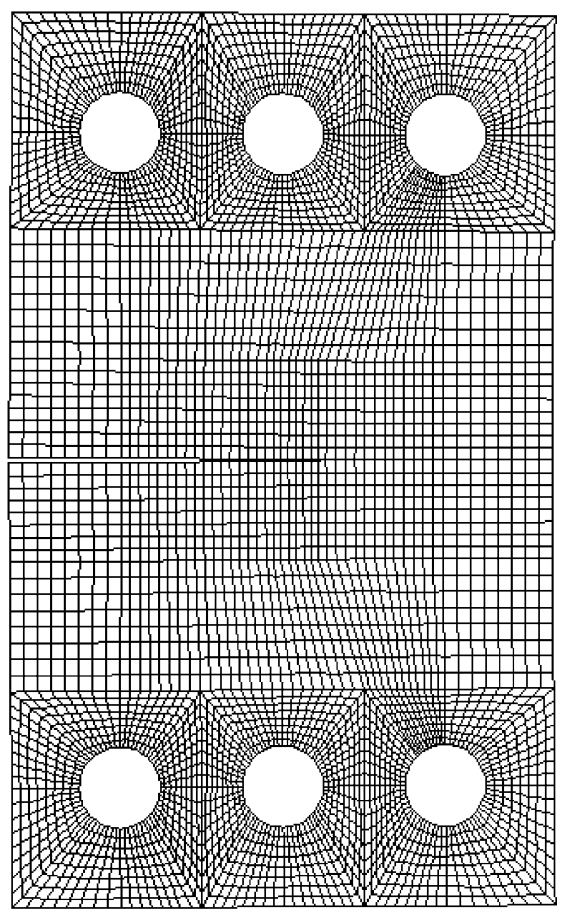

(b)

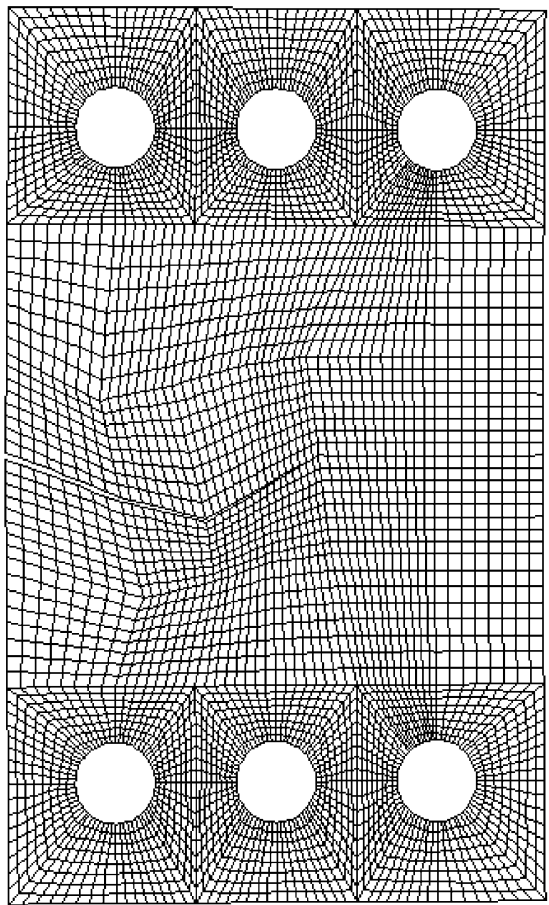

Fig. 5. Finite element mesh for different slops at the crack tip: (a) $\beta=0,60^{\circ}$.

To evaluate the characteristics of mixed mode fatigue crack, it is necessary to introduce the comparative stress intensity factor $K_{\mathrm{V}}$ considering mode I and mode II simultaneously. The exact relation between $K_{\mathrm{V}}, K_{\mathrm{I}}$ and $K_{\mathrm{II}}$ depends on the criterion employed. Accordingly to Richard/Henn criterion that relation is $[1,16]$

$K_{\mathrm{V}}=\frac{K_{\mathrm{I}}}{2}+\frac{1}{2} \sqrt{K_{\mathrm{I}}^{2}+6 K_{\mathrm{II}}^{2}}$ 
Furthermore, the comparative stress intensity factor range, $\Delta K_{\mathrm{V}}$, is

$\Delta K_{\mathrm{V}}=\frac{\Delta K_{\mathrm{I}}}{2}+\frac{1}{2} \sqrt{\Delta K_{\mathrm{I}}^{2}+6 \Delta K_{\mathrm{II}}^{2}}$

where $\Delta K_{\mathrm{I}}$, and $\Delta K_{\mathrm{II}}$ are the mode-I and mode-II stress intensity factor ranges, respectively.

In this work the maximum and minimum loads applied for each loading angle were chosen in order to have after fatigue pre-crack a comparative stress intensity factor, $\Delta K_{\mathrm{V}}$, of approximately $6 \mathrm{MPa} \mathrm{m}{ }^{1 / 2}$, as given by Eq. (7).

\section{Results and discussion}

\subsection{Experimental analysis}

Fig. 6 shows crack growth paths for several loading angles. It was observed that fatigue crack growth direction changed immediately from the initial notch and fatigue mode-I precrack orientation when load direction changed. The initial slopes at the crack tip were $\beta=29.56,41.50$ and $54.30^{\circ}$ for loading angles of $\alpha=30,45$ and $60^{\circ}$, respectively. In all cases $\beta$ decreased with cyclic loading.

Fig. 7 shows the fatigue crack growth behaviour under mixed-mode $\left(\alpha=30,45\right.$ and $\left.60^{\circ}\right)$ and mode-I loads obtained from the comparative stress intensity factor range defined in Eq. (7).

It can be clearly seen that $\Delta K_{\mathrm{V}}$ is able to correlate satisfactorily the influence of the mixed-mode loading conditions and mode-I loading for low $\Delta K_{\mathrm{V}}$ values below approximately $10 \mathrm{MPa} \mathrm{m}^{1 / 2}$, corresponding to crack growth rates in the range $10^{5}-10^{4} \mathrm{~mm} /$ cycle. For $\Delta K_{\mathrm{V}}$ values higher than $10 \mathrm{MPa} \mathrm{m}{ }^{1 / 2}$ the growth rates of cracks that were grown under mixed-mode loading cannot be correlated further with crack growth rate under mode-I loading by the comparative stress intensity factor range as given by Eq. (7). Furthermore, the difference between the crack growth rates under mixed-

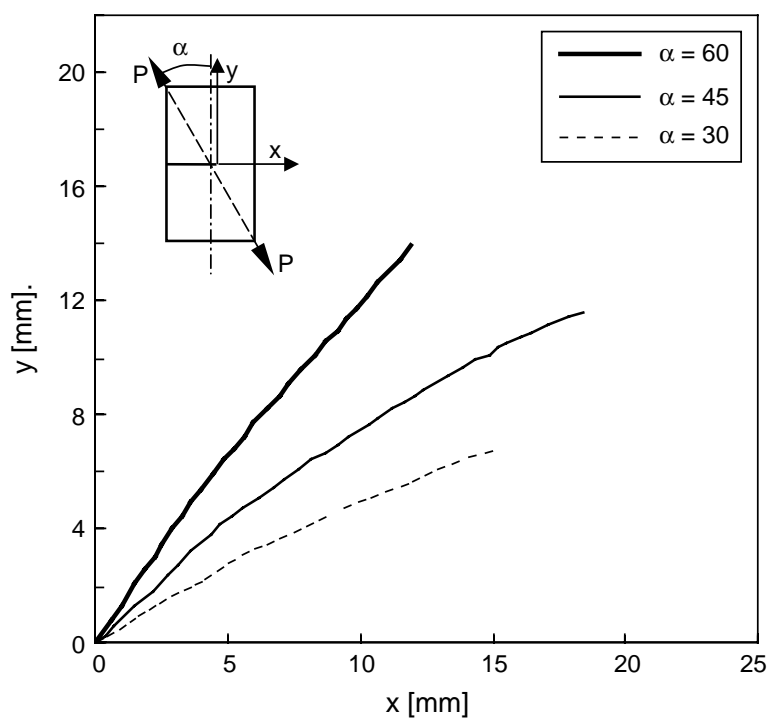

Fig. 6. Crack growth paths for several loading angles.

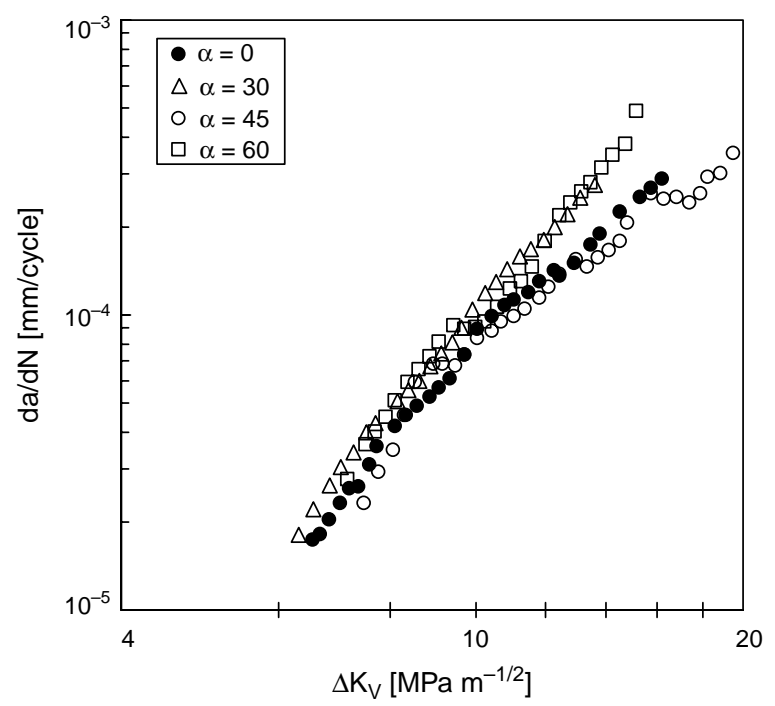

Fig. 7. Crack growth rate versus the comparative stress intensity factor $\Delta \mathrm{K}_{V}$.

mode and mode-I loadings increases with $\Delta K_{\mathrm{V}}$ Similar results were obtained in ref. [4] in a rail steel under mixed-mode loadings.

Crack closure levels for mode-I loading as well as for the mixed-mode loading conditions are presented in Fig. 8 by plotting the normalised load ratio parameter $U$, defined by Eq. (1), against the crack length.

This figure shows that in general $U$ decreases, i.e. the crack closure level increases for mixed-mode loading conditions in comparison to mode-I loading. Moreover, crack closure only increases slightly for $\alpha=30^{\circ}$ while for loading angles of $45^{\circ}$ and $60^{\circ}$ increases significantly. For low crack lengths parameter $U$ drops steeply with decreasing crack length. Such behaviour is generally associated to the dominance of roughness-induced crack closure $[8,10]$. Above $a=54 \mathrm{~mm}$ parameter $U$ practically stabilises between the values of 0.87 and 0.91 for mode-I and mixed-mode loadings with $\alpha=30^{\circ}$. For loading angles of $45^{\circ}$ and $60^{\circ}$ this condition was not attained. For higher crack lengths plasticity-induced crack closure probably dominates and the closure level is generally independent of $\Delta K$.

For the same comparative stress intensity factor, $\Delta K_{\mathrm{V}}$, the $K_{\mathrm{I}}$ component decreases with increasing loading angle, $\alpha$. Furthermore, the fatigue crack growth path under mixed-mode is more tortuous than that under mode I loading. Therefore, the occurrence of asperity contact is promoted under mixed-mode loading, which enhances roughness-induced closure. This results in an increase of the crack opening load, which implies a reduction of the minimum effective driving force behind the crack.

Fig. 9 shows the $\mathrm{d} a / \mathrm{d} N-\Delta K_{\text {eff }}$ data for mixed-mode and mode-I loading conditions. The change of effective stress intensity factor $\Delta K_{\text {eff }}$ was calculated from $U$ and $\Delta K$ according to the following equation:

$\Delta K_{\mathrm{eff}}=\frac{U \Delta K_{\mathrm{I}}}{2}+\frac{1}{2} \sqrt{\left(U \Delta K_{\mathrm{I}}\right)^{2}+6 \Delta K_{\mathrm{II}}^{2}}$ 


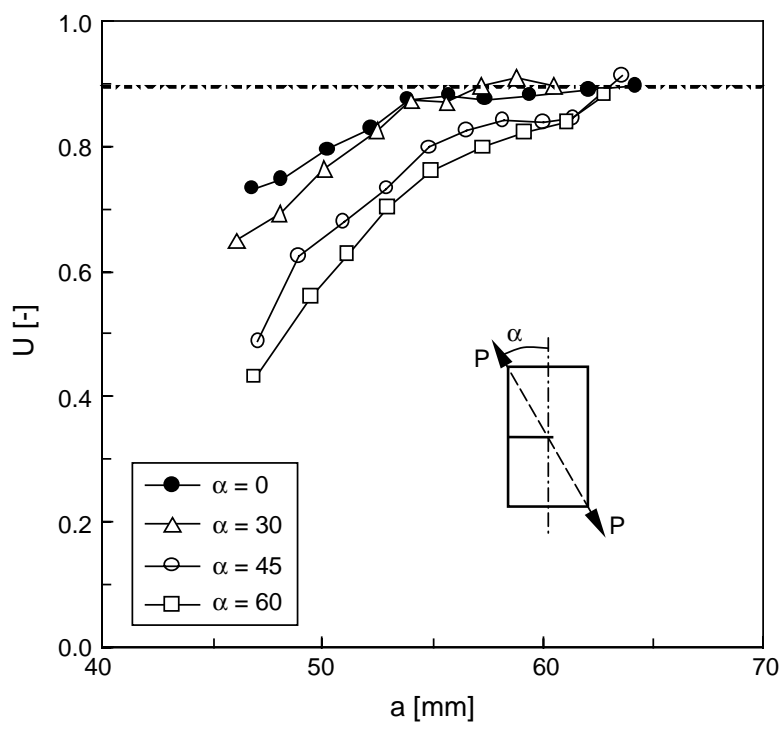

Fig. 8. Crack closure levels.

This figure shows that the fatigue crack growth rate data tend to fall within a very narrow scatter band when $\mathrm{d} a / \mathrm{d} N$ is plotted against $\Delta K_{\text {eff }}$ calculated accordingly to Eq. (8). Therefore, crack closure permits the reduction of all the crack growth rate data to a unique curve $\mathrm{d} a / \mathrm{d} N-\Delta \mathrm{K}_{\text {eff }}$ once the crack closure effect is accounted for.

\subsection{Elastic-plastic finite element analysis}

An elastic-plastic finite element analysis was also done in order to obtain the plastic zones sizes and shapes and model the effect of both modes on crack closure induced by plasticity. The physical model was analysed using MARC, a commercial finite element package [17], and the procedure initially established for mode-I loading [12]. The elastic-plastic behaviour was studied assuming Von Mises yield criterion, incremental Prandtl-Reuss plasticity rule, large strains and

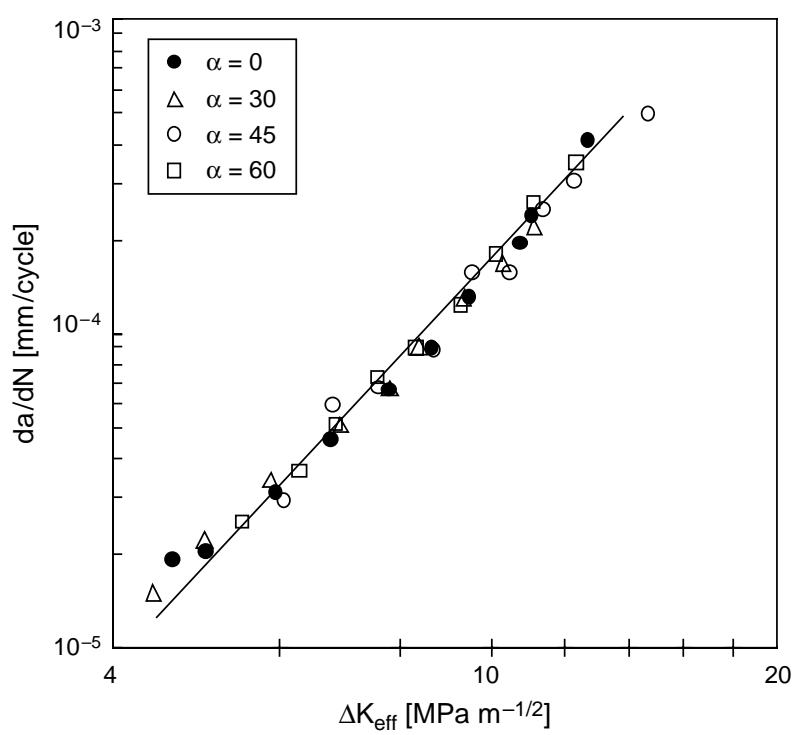

Fig. 9. Crack growth rate versus the effective stress intensity factor $\Delta K_{\text {eff }}$. large displacements. The back-stress of kinematic hardening rule was defined by Ziegler's hardening rule. The cyclic stressstrain curve, obtained experimentally [18], was used. Quadrilateral isoparametric elements with eight nodes were considered. A comparative stress intensity factor range (Eq. (7)) of $9 \mathrm{MPa} \mathrm{m}^{1 / 2}$ was chosen for this analysis. Fig. 10 presents the finite element mesh having elements with $8 \mu \mathrm{m}$ near the crack tip. Due to lack of symmetry in terms of loading, the whole specimen needs to be analysed. Square elements were used at the crack tip to minimise interpolation errors and so maximise convergence rate. The entire mesh included about 6700 elements and 20687 nodes.

(a)

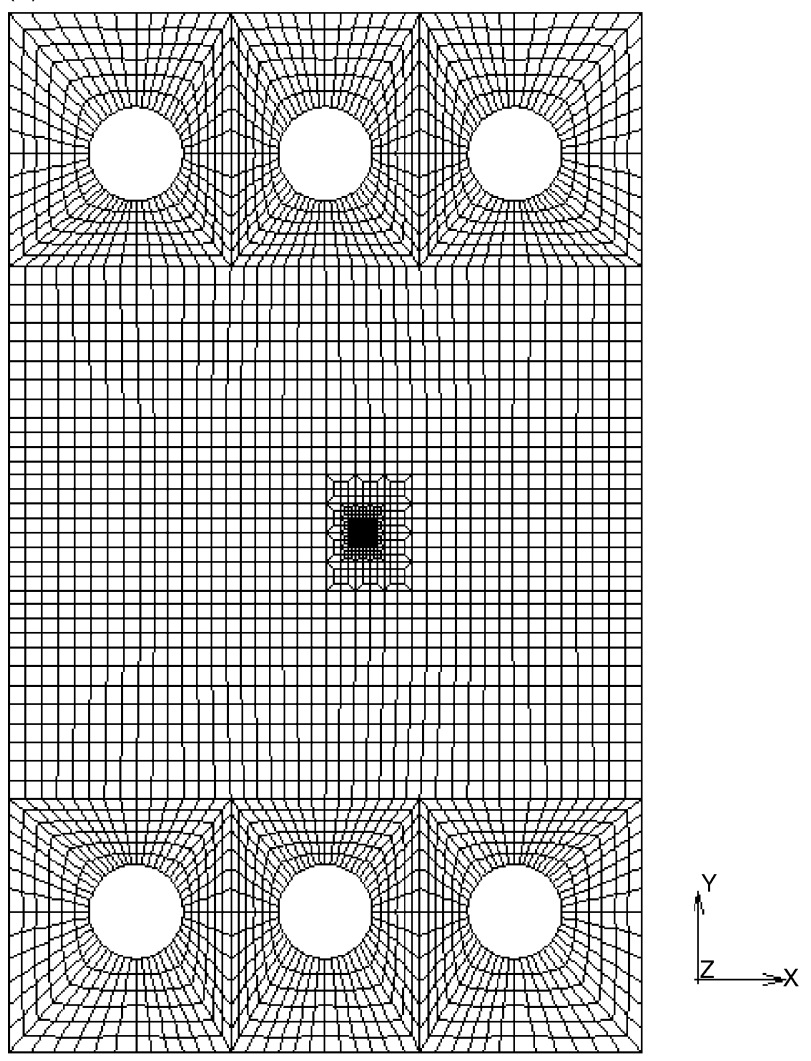

(b)

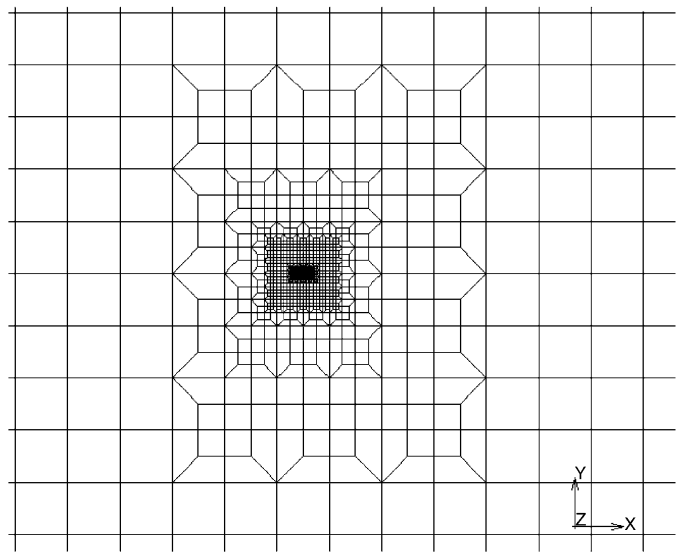

Fig. 10. Finite element mesh of CTS specimen for elastic-plastic behaviour: (a) whole mesh, (b) detail near the crack tip. 


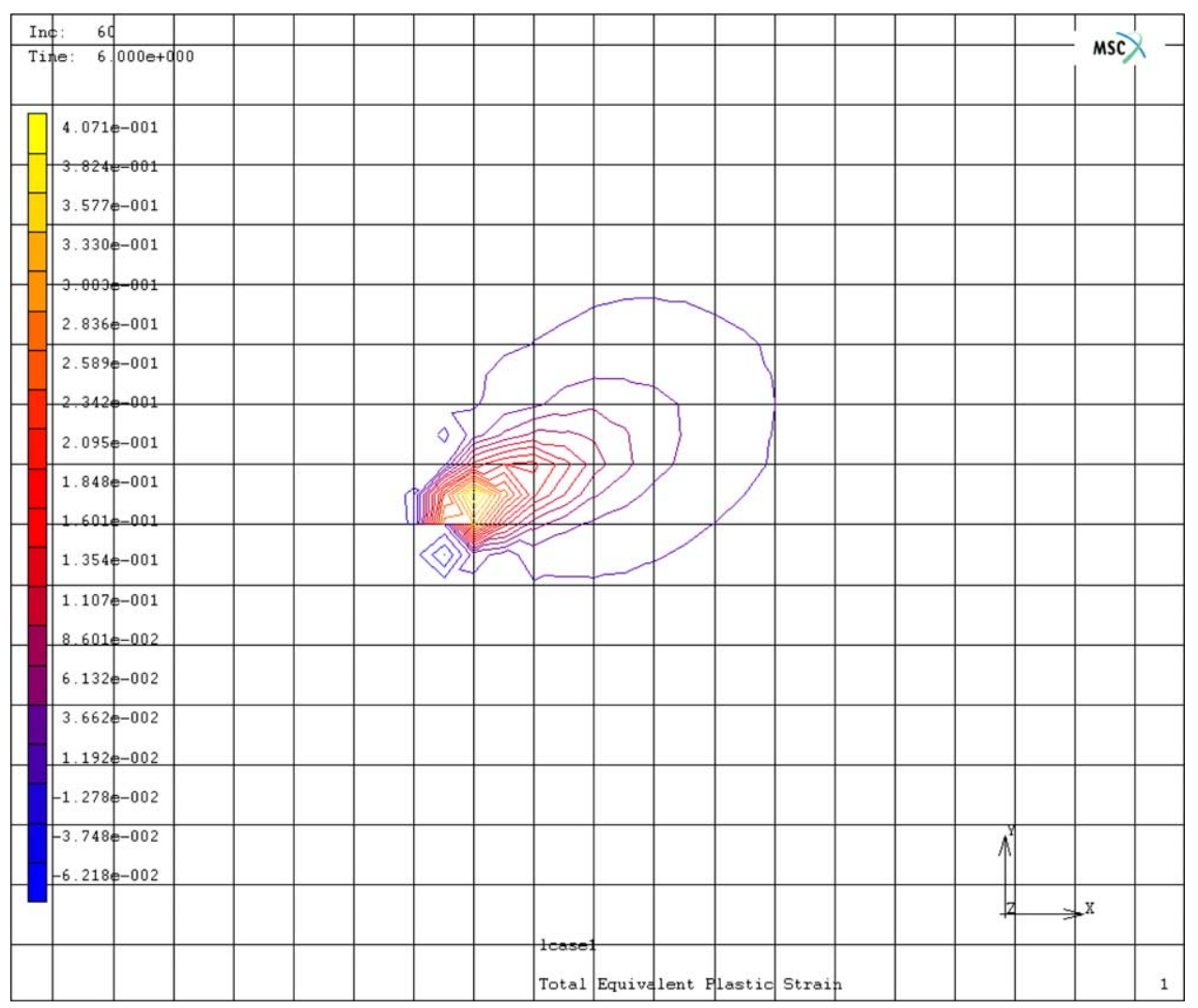

Fig. 11. Total equivalent plastic strain at load direction angle $\alpha=30^{\circ}$ and $\Delta K_{\mathrm{V}}=6 \mathrm{MPa} \mathrm{m}^{1 / 2}$.

Iteration to the correct solution of the nonlinear equations at each load step was carried out according to the NewtonRaphson method. The iterative procedure was terminated when the ratio maximum residual force/maximum reaction force was smaller than the convergence limit. Crack extension was achieved changing contact conditions and each crack increment had usually a value $\Delta a^{*}$ equal to the size of crack tip elements. Crack propagation was done at maximum load, similarly as for pure mode-I loading [12]. Last contact of nodes behind crack tip with a rigid line positioned on the crack plane was considered as definition of opening level. In order to obtain accurate opening and closure values, a relatively large number of steps (80) was used.

The finite element results indicate that the size of monotonic plastic zones does not vary significantly with loading direction. On the other hand plastic strain distribution varies significantly. The increase of load direction angle $(\alpha)$ rotates the direction of maximum size of plastic zone size, which is always normal to load direction, as depicted in Fig. 11 at $\alpha=30^{\circ}$ and $\Delta K_{\mathrm{V}}=$ $6 \mathrm{MPa} \mathrm{m}^{1 / 2}$.

Table 3 presents the comparison between the elastic-plastic finite element results and measured crack closure levels under mode-I $\left(\alpha=0^{\circ}\right)$ and mixed-mode loading at load direction angles of $30^{\circ}$ and $60^{\circ}$. This comparison is also depicted in Fig. 12. In this figure the crack closure levels obtained numerically are indicated by horizontal lines because the elastic-plastic finite element analysis produces stable values of closure as it only simulates plasticity-induced closure.
Numerical closure levels practically independent of $\Delta K$ were also observed for Middle-Tension specimens under mode-I loading at medium and high $\Delta K$ values [12].

In all cases higher $U$ values, i.e. lower crack closure levels, were obtained numerically in comparison to experimental values. However, for mode-I and $\alpha=30^{\circ}$ the numerical values are similar to the stable crack closure levels obtained experimentally, with differences lower than 2.2 and $5.4 \%$, respectively, which is consistent with the dominance of the plasticity-induced crack closure mechanism at medium and high $\Delta K$ values. Contrary to the observed for these loading conditions, at lower $K_{\mathrm{I}} / K_{\mathrm{II}}$ ratios $\left(\alpha=60^{\circ}\right)$, significantly lower crack closure levels (up to $22 \%$ ) are obtained by the elasticplastic finite element analysis in comparison with measured values at medium $\Delta K$ values.

The behaviour observed under mode-I and $\alpha=30^{\circ}$ can be considered very reasonable considering the limitations of the physical model, namely, in terms of material behaviour and simulation of crack growth. Wei and Ellyin [19] studied a CT

Table 3

Measured and numerical crack closure levels under mode-I and mixed-mode loading at $\Delta K_{\mathrm{V}}=9 \mathrm{MPa} \mathrm{m}^{1 / 2}$

\begin{tabular}{llll}
\hline Loading & Mode-I & \multicolumn{2}{l}{ Mixed-mode (I+II) } \\
\cline { 2 - 4 } & $\alpha=0^{\circ}$ & $\alpha=30^{\circ}$ & $\alpha=60^{\circ}$ \\
\hline Experimental & $U=0.88$ & $U=0.87$ & $U=0.74$ \\
Numerical & $U=0.90$ & $U=0.92$ & $U=0.95$ \\
\hline
\end{tabular}




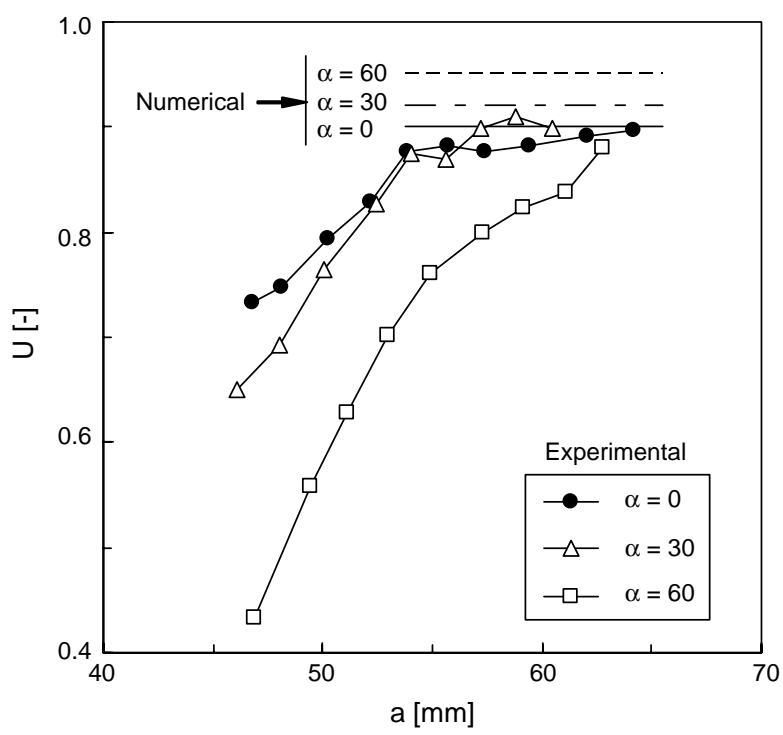

Fig. 12. Comparison between experimental measurements and numerical crack closure levels.

specimen with $10 \mathrm{~mm}$ thickness under mode-I loading and found differences within $10-15 \%$ when plane stress numerical data were compared with near-tip strain gauge output and plane strain results were compared with back face strain values.

It is important to note that the numerical analysis only intends to simulate plasticity-induced closure. In spite of expecting the dominance of this type of closure mechanism after $9 \mathrm{MPa} \mathrm{m}{ }^{1 / 2}$, the occurrence of some roughness and oxide induced closure can not be completely discharged, even under mode-I loading. As already mentioned, at lower $K_{\mathrm{I}} / K_{\mathrm{II}}$ ratios the occurrence of asperity contact is promoted under mixedmode loading which enhances roughness-induced closure, therefore the differences between numerical and experimental crack closure levels are expected to increase as indeed observed. Furthermore, other limitations of the numerical analysis can also contribute to these differences, namely:

(i) the experimental cyclic stress-strain curve had a limited extent. Therefore, since important deformations are obtained at the crack tip an extrapolation was needed, which can introduce errors;

(ii) an isotropic behaviour is assumed for the material, however real specimens were obtained from laminated plates and can have anisotropic behaviour;

(iii) according to striation model of crack growth propagation is not a sudden process occurring at a defined load but a continuous process. Besides, crack increments considered are much higher than real values.

\section{Conclusions}

From the experimental and numerical study on fatigue crack growth and crack closure behaviours in AlMgSi1-T6 aluminium alloy under mixed-mode loading at various $K_{\mathrm{I}} / K_{\mathrm{II}}$ ratios, the following conclusions can be drawn:
1. Fatigue crack growth direction changes immediately from the initial notch orientation when load direction is modified. Furthermore, the crack tip angle $\beta$ decreases with cyclic loading.

2. The comparative stress intensity factor range $\Delta \mathrm{K}_{\mathrm{V}}$ correlates satisfactorily the influence of the mixed-mode loading conditions and mode-I loading only for low $\Delta \mathrm{K}_{\mathrm{V}}$ values.

3. Crack closure increases under mixed-mode loading conditions in comparison to mode-I loading. Moreover, the crack closure level increases with the $\mathrm{K}_{\mathrm{I}} / \mathrm{K}_{\mathrm{II}}$ ratio decrease.

4. Roughness-induced closure is promoted under mixed-mode loading due to the occurrence of enhanced asperity contact, which implies a reduction of the minimum effective driving force behind the crack.

5. Significantly lower crack closure levels are obtained by the elastic-plastic finite element analysis relatively to measured values at high load direction angles (low $\mathrm{K}_{\mathrm{I}} / \mathrm{K}_{\mathrm{II}}$ ) mainly due to the occurrence of roughness-induced closure.

\section{Acknowledgements}

The authors would like to acknowledge POCTI programme, project EME/47022/2002, for funding the work reported.

\section{References}

[1] Richard HA, Linnig W, Henn K. Fatigue crack propagation under combined loading. Forensic Eng 1991;3:99-109.

[2] Biner SB. Fatigue crack growth studies under mixed-mode loading. Int J Fatigue 2001;23(Suppl.):S259-S63.

[3] Sander M, Richard HA. Lifetime predictions for real loading situationsconcepts and experimental results of fatigue crack growth. Int J Fatigue 2003;25:999-1005.

[4] Kim J-K, Kim C-S. Fatigue crack growth behaviour of rail steel under mode I and mixed mode loadings. Mater Sci Eng 2002;A338:191-201.

[5] Abdel Mageed AM, Pandey RK. Studies on cyclic crack path and the mixed-mode crack closure behaviour in Al alloy. Int J Fatigue 1992;14: 21-9.

[6] Blom AF, Holm DK. An experimental and numerical study of crack closure. Eng Fract Mech 1984;22:997-1011.

[7] Paris PC, Tada H, Donald JK. Service load fatigue damage-a historical perspective. Int J Fatigue 1999;21(Suppl.):S35-S46.

[8] Borrego LP, Ferreira JM, Costa JM. Fatigue crack growth and crack closure in an AlMgSi alloy. Fatigue Fract Eng Mater Struct 2001;24: 255-65.

[9] Bergner F, Zouhar G, Tempus G. The material-dependent variability of fatigue crack growth rates of aluminium alloys in the Paris regime. Int J Fatigue 2001;23:383-94.

[10] Borrego LP, Costa JM, Ferreira JM. Microstructure dependent fatigue crack growth in aged hardened aluminium alloys. Int J Fatigue 2004;26: 1321-31.

[11] Borrego LP, Ferreira JM, Pinho da Cruz JM, Costa JM. Evaluation of overload effects on fatigue crack growth and closure. Eng Fract Mech 2003;70:1379-97.

[12] Antunes FV, Borrego LP, Costa JM, Ferreira JM. A numerical study of fatigue crack closure induced by plasticity. Fatigue Fract Eng Mater Struct 2004;27:825-36. 
[13] Richard HA. Bruchvorhersagen bei überlagerter normal- und schubbeanspruchung von risen VDI Forschungsheft 631. Düsseldorf: VDI-Verlag; 1985. p. 1-60.

[14] Allison JE, Ku RC, Pompetzki MA. A comparison of measurement methods and numerical procedures for the experimental characterization of fatigue crack closure. In: Newman Jr JC, Elber W, editors. Mechanics of fatigue crack closure, ASTM STP 982. Philadelphia, PA: ASTM 1988. p. 171-85.

[15] Cosmos/M manuals Los Angeles, CA: Structural Research and Analysis Corporation (SRAC) 2000.
[16] Henn K, Richard HA, Linnig W. Fatigue crack growth under mixed mode and mode II cyclic loading. In: Czoboly E, editor. Fracture analysistheory and practice, vol. II. Warley (UK): EMAS-Ltd; 1988. p. 1104-13.

[17] MARC User Information. Palo Alto: Marc Analysis Research Corp.; 2000.

[18] Borrego LP, Abreu LM, Costa JM, Ferreira JM. Analysis of low cycle fatigue in AlMgSi aluminium alloys. Eng Fail Anal 2004;11:715-25.

[19] Wu J, Ellyin F. A study of fatigue crack closure by elastic-plastic finite element analysis for constant-amplitude loading. Int J Fract 1996;82: $43-65$. 